\title{
Exercise-induced Hypoalgesia Profiles for Pain Prediction in the Trigeminal System
}

\section{Khalil Assiri}

\section{ABSTRACT}

Aim: This study aimed to evaluate the predictivity of exerciseinduced hypoalgesia $(E I H)$ profile on pain severity produced by nerve injury in an animal model.

Materials and methods: A total of 51 rats were divided according to the EIH model, which was evaluated by the percentage of withdrawal actions of 30 repetitive physical stimuli on the hind paw of the rat before and after 3 minutes of exercise on a treadmill, into high and low EIH. Left infraorbital nerve injury was then done on rats from high and low EIH groups. Pain assays as mechanical allodynia and mechanical hyperalgesia were evaluated in the ipsilateral and unaffected whisker pad before and 3,10 , and 17 days after the surgery.

Results: Rats that were categorized as low EIH showed increased hypersensitivity and demonstrated significantly severe mechanical hyperalgesia in ipsilateral side 10 and 17 days after the surgery compared with the high EIH rats.

Conclusion: The authors concluded that the pinprick behavioral assay (mechanical hyperalgesia) was able to predict chronic pain development in the area of the trigeminal nerve distribution using EIH profile measured at the hind paw of the rat.

Keywords: Exercise-induced hypoalgesia, Nerve injury, Neuropathic pain.

How to cite this article: Assiri K. Exercise-induced Hypoalgesia Profiles for Pain Prediction in the Trigeminal System. World J Dent 2017;8(1):21-27.

Source of support: Nil

Conflict of interest: None

\section{INTRODUCTION}

Pain according to the International Association for the Study of Pain (IASP) is defined as "an unpleasant sensory and emotional experience associated with actual or potential

\footnotetext{
Assistant Professor

Department of Oral and Maxillofacial Pathology, Radiology and Medicine, NYU Orofacial and Head Pain Service, New York University College of Dentistry, New York, USA; Department of Diagnostic Sciences, Rutgers School of Dental Medicine Newark, New Jersey, USA; Department of Diagnostic Sciences College of Dentistry, King Khalid University, Abha, Kingdom of Saudi Arabia

Corresponding Author: Khalil Assiri, Assistant Professor Department of Oral and Maxillofacial Pathology, Radiology and Medicine, NYU Orofacial and Head Pain Service, New York University College of Dentistry, New York, USA; Department of Diagnostic Sciences Rutgers School of Dental Medicine, Newark New Jersey, USA; Department of Diagnostic Sciences, College of Dentistry, King Khalid University, Abha, Kingdom of Saudi Arabia
}

tissue damage, or described in terms of such damage." The process of pain includes the following steps:

- Transduction: Conversion of a noxious stimuli to an electrical impulse referred to as an action potential.

- Transmission: Process by which the action potential will be transferred from the periphery to the nervous system.

- Modulation: This process involves the modification of the pain stimuli either by facilitating or inhibiting it depending upon the environment.

- Perception: In this stage, the nerve impulses finally reach the somatosensory cortex of the brain and will be perceived as pain. ${ }^{1}$

Orofacial pain can be classified according to the etiology into three different categories: ${ }^{2}$

1. Musculoskeletal

2. Neuropathic

3. Neurovascular

Neuropathic pain (NP) is defined as a condition initiated or caused by a primary lesion or dysfunction in the nervous system. ${ }^{3}$ Although the exact mechanism for the development and maintenance of NP is not fully understood, a complex interaction between the nervous system and the immune system has been suggested. ${ }^{4,5}$ One of the causes for NP is nerve damage. There are different types of nerve damage that can occur. One of them is transection in which there is a total loss of nerve fiber continuity. Clinically, it can be demonstrated in surgical cases. Another type of nerve injury is crush where there is a partial loss of nerve fiber with some level of continuity. Clinically, it is observed in fractures and trauma injuries. A third type is pressure, e.g., chronic pressure by a tumor or a benign lesion, such as in carpel tunnel syndrome or squamous cell carcinoma of the face or neck. Finally, inflammation can also cause direct or indirect nerve damage and can be seen in cases of dental implants, which have been placed too close to a nerve leading to perineural or nerve inflammation. It is observed especially in cases where the dental implant is in close proximity to the inferior alveolar nerve canal. Various qualities and sensations have been described in patients experiencing NP. Some of these have been defined by the IASP as follows:

Hyperalgesia: "Increased pain from a stimulus that normally provokes pain"

- Primary hyperalgesia - occurs at site of injury

- Secondary hyperalgesia - occurs in the central nervous system 
Allodynia: "Pain due to a stimulus that does not normally provoke pain"

Activation of A-beta (touch) fibers can cause pain

Anesthesia dolorosa: Pain in an area or region which is anesthetic

Paresthesia: "An abnormal sensation, whether spontaneous or evoked"

Dysesthesia: "An unpleasant abnormal sensation, whether spontaneous or evoked".

At present, NP is treated primarily by pharmacotherapy on a trial and error approach. The current treatments can achieve partial pain relief; however, it can accompany significant side effects. Other treatment options may include surgery, psychological intervention, and adjunctive therapy, such as acupuncture, yoga, meditation, biofeedback hypnosis, and exercise.

Exercise is commonly used as part of treatment and rehabilitation for chronic pain patients. Conditions like fibromyalgia, which is characterized by global muscle and joint pain, muscle pain, arthritis, and NP, have shown therapeutic analgesic effects to exercise. ${ }^{6}$ Both human and animal models have validated these findings. Inhibitory neurotransmitters, such as serotonin and dopamine have been linked to an elevation postexercise sessions. ${ }^{6-8}$ The exact mechanism of exercise-induced analgesia is poorly understood. Studies have shown that after aerobic exercise, there is an analgesic effect to thermal and mechanical stimuli. ${ }^{9,10}$ However, the endocannabinoid system is widely accepted as a fundamental factor in this hypoalgesic response. ${ }^{11}$ Preliminary studies have shown that blocking of the endocannabinoid system cannot only alter the exercise-induced hypoalgesia (EIH) but also result in lower pain thresholds. ${ }^{11}$ Previous studies have also shown that submaximal isometric contraction of muscle can cause a significant increase in pain pressure threshold in the contracted muscle, contralateral resting muscles, and distant resting muscle. This suggests a generalized modulation effect of the pain mechanisms. ${ }^{12}$ Studies have shown that swimming can significantly reduce $\mathrm{NP}^{13,14}$ and reduce mechanical allodynia and thermal hyperalgesia induced by chronic constriction nerve injury (CCI) in rat model of nerve injury. ${ }^{15}$ Also, high-intensity swimming exercise can reduce NP in an animal model of complex regional pain syndrome type I. ${ }^{16}$ Another study showed that regular physical activity prevents development of chronic pain and activation of central neurons, ${ }_{1}^{17}$ while other studies show that experimentally induced pain perception is acutely reduced by aerobic exercise in people with chronic low back pain. ${ }^{18}$ Recent studies have shown that EIH profiling has the ability to predict acute and chronic NP in the sciatic nerve model of a rat. ${ }^{19}$

The model used for profiling EIH included 30 repetitive noxious stimuli with a $60 \mathrm{gm}$ von Frey filament on the hind paw of rat pre- and post-180 seconds of exercise. A percentage change in the response to the noxious stimuli postexercise was used to categorize the rats into a high, medium, or low EIH group. A response change of 0 to $33.3 \%$ put them in the low EIH group, whereas a response change of 33.3 to 66.6 and 66.6 to $100 \%$ put them in the medium and high EIH group respectively. When these rats were subjected to nerve injury, the rats with a high EIH profile developed significantly less NP as compared with the low EIH group. These results indicate that a baseline (BL) profiling before injury can actually predict the pain developed. It can be of great clinical importance, as it will help us understand not only the mechanism of EIH but also which patients are more prone to develop pain postsurgery. In this study, we wanted to understand if the EIH profiling can predict pain in the orofacial region following nerve damage to the infraorbital nerve of the rat. This will help us understand if the EIH has a localized effect or a more global effect in predicting pain. The EIH will be performed on the hind paw of the rat, whereas the nerve damage will be done on the infraorbital nerve of the face.

\section{MATERIALS AND METHODS}

All experimental protocols were in compliance with the guidelines of IASP. Adult male Sprague Dawley rats weighing 250 to $300 \mathrm{gm}$ were used in this study. Rats were habituated preoperatively by allowing them 10 to 20 minutes in the sensory testing apparatus for five consecutive days. During this period, rats were tested for tactile allodynia in the region of the infraorbital nerve. During the entire period, they were maintained on standard mouse chow, reverse osmotically treated water, and also a 12-hour day and night cycle.

\section{Phase I}

In the first phase, EIH was performed on healthy rats. The EIH was measured using a model previously used. In the model, temporal summation was measured by applying 30 repetitive stimuli with a $60 \mathrm{gm}$ von Frey filament to the hind paw of the rat. The rats were then allowed to exercise on a treadmill (Rota Rod) for 3 minutes. Immediately after the exercise within 1 minute, the response to the noxious stimuli (von Frey filament) was reassessed. Depending upon the change of response from BL, the rats were categorized into high, medium, and low EIH rats. Rats that have a change of above $66 \%$ were considered high EIH, rats with a response change from 33.3 to $64 \%$ were considered medium $\mathrm{EIH}$, and rats with a response change of $33 \%$ or below were considered low EIH rats. In brief, in the first phase of the study, EIH was measured and rats were grouped into high, medium, and low EIH 
groups. In this study, we included only the high and low EIH groups.

\section{Phase II (Surgical Phase)}

\section{Anesthesia}

For surgical procedures, rats were anesthetized with ketamine $(50 \mathrm{mg} / \mathrm{kg})$ and xylazine $(7.5 \mathrm{mg} / \mathrm{kg})$ solution.

\section{Surgery}

Chronic constriction injury surgery was performed based on the original model developed by Imamura et al. ${ }^{20}$ In brief, about $1-\mathrm{cm}-$ long incision was done on the area of the gingivobuccal margin mesial to the first molar. Approximately $0.5 \mathrm{~cm}$ of the infraorbital nerve (ION) was exposed, and then two chromic gut knots were done loosely around the nerve.

Previous studies have shown that rats with high EIH develop less NP after sciatic nerve damage compared with low EIH rats. ${ }^{19}$ This model has the ability to predict the pain developed in both the acute and chronic phases of NP. We used the same model of EIH, but the surgery was performed in the infraorbital nerve of the face. This allowed us to understand if the predictive effect of EIH is global or localized to the sciatic nerve injury. Various studies have shown that the trigeminal and nontrigeminal response to noxious stimuli is different. Thus, we will be able to understand the effect of EIH on a broader scale.

\section{PAIN BEHAVIOR ASSAY}

\section{Tactile Allodynia}

Tactile allodynia was tested with von Frey fibers using Semmes-Weinstein monofilaments sorted by ranks expressing the logbase10 of the force applied in milligrams to bend the filament (Stoelting, Wood Dale, IL, USA). To evaluate tactile allodynia, the rats' withdrawal response to an increasing amount of force delivered by a calibrated von Frey filament was measured. The monofilaments were applied in the left rat's infraorbital nerve region extraorally. The test was performed with the rat inside a chamber placed on an elevated perforated floor. Fibers were applied in an ascending order to detect the threshold. Typically, rats in pain respond to a lower force as compared with nonpainful rats.

\section{Mechanical Hyperalgesia}

Mechanical hyperalgesia was tested with pinprick stimulation. The blunt acupuncture needle was used in the area of whisker pad, until the skin was pitted without penetration. Then the ordinal scale of rat's response was used as follows: No detection $=0$, response $=1$, withdrawal action after response $=2$, withdrawal and aggressive actions after detection $=3$, same as 3 but with facial grooming $=4 .{ }^{21}$

\section{SAMPLE SIZE AND STATISTICAL ANALYSIS}

Alpha (two tailed) for significance in all analysis was set at 0.05 . A confidence interval of $95 \%$ was set. Sample size analysis required eight animals per group to get a power of $80 \%$. Data were tabulated and analyzed using StatView 5 software (SAS Institute Inc., NC, USA). Behavioral statistics were performed only on rats with data at all time points. For tactile allodynia, time points of relevance were analyzed with a repeated measures analysis of variance (ANOVA). Within group, data were compared with repeated measures ANOVA. For tactile allodynia, results were expressed as force to which response was recorded ( $\log 10 \mathrm{gm} \pm$ standard error of the mean).

\section{RESULTS}

A total of 51 rats were included in the study: All rats underwent habituation, EIH evaluation, nerve injury, and pain behavior assessment.

\section{Phase I: EIH Assessment}

The percentage change from BL response to 1-minute response was calculated for each rat, such that

$$
\mathrm{EIH}=\frac{\mathrm{BL} \text { Response }-1 \text { minute respose }}{\mathrm{BL} \text { Response }} \times 100
$$

Based on this equation, 30 rats were considered as high EIH (reduction of $66.67 \%$ or more to response compared with BL). The low EIH profile included 21 rats (reduction of $33.33 \%$ or less to response compared with BL).

\section{Phase II: Pain Behavior}

The rats' pain behaviors were assessed prior to the surgery and 3, 10, and 17 days following the surgery. The high $(n=30)$ and low $(n=21)$ EIH groups' pain behavior scores were compared at each time point.

\section{Mechanical Hyperalgesia}

Ipsilateral side: No significance difference was found between low and high EIH rats at BL $(1.238 \pm 0.118$, $1.033 \pm 0.089, \mathrm{p}>0.05)$. At day 3 postsurgery, no significant difference was found between low and high EIH rats $(2.238 \pm 0.194,2.100 \pm 0.194, \mathrm{p}>0.05)$. At day 10 postsurgery, there was a significant difference between the low and high EIH profile rats in pain behavior $(3.095 \pm 0.275$, $2.367 \pm 0.237, \mathrm{p}<0.05)$. At day 17 postsurgery, there is also a significant difference between low and high EIH profile rats $(2.952 \pm 0.223,2.115 \pm 0.231, \mathrm{p}<0.05)($ Graph 1$)$. 


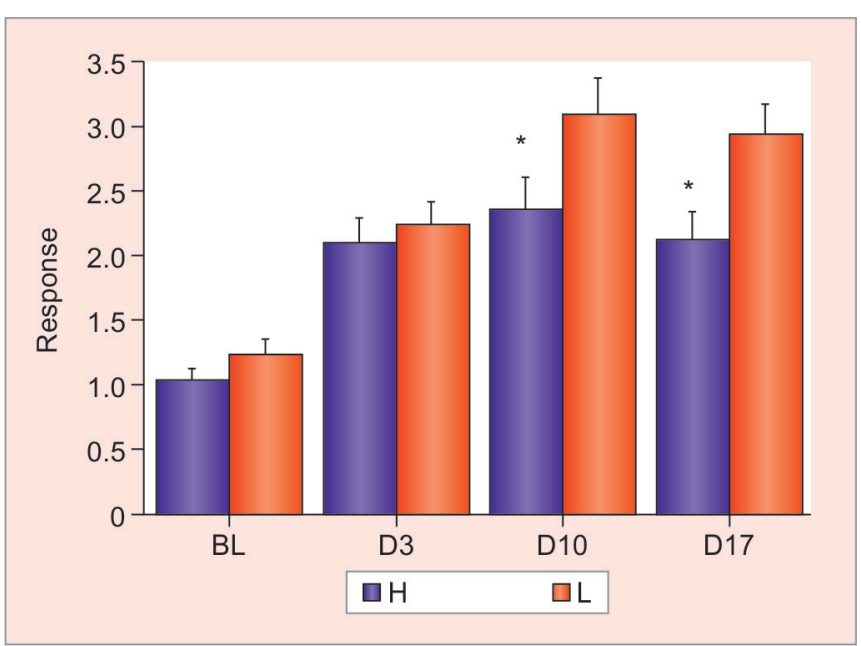

Graph 1: Mechanical hyperalgesia response: Ipsilateral side, $\mathrm{H}=$ high $\mathrm{EIH}$ group, $\mathrm{L}=$ low EIH group, $\mathrm{BL}=$ baseline, $\mathrm{D} 3=$ day 3, D10 $=$ day $10, \mathrm{D} 17=$ day $17,{ }^{*}$ It means there is a statistically significant difference between the two groups in the response. $p<0.05$ )

Contralateral side: No significant difference was found between low and high EIH rats at BL, day 3, day 10, and day $17[(0.273 \pm 0.104,0.385 \pm 0.162, \mathrm{p}>0.05)$, $(0.273 \pm 0.124,0.308 \pm 0.106, \mathrm{p}>0.05),(0.318 \pm-0.139,0.231+$ $-0.108, p>0.05),(0.250+-0.171,0.300+-0.153, p>0.05)]$ (Graph 2).

\section{Mechanical Allodynia}

Ipsilateral side: No significant difference was found between low and high EIH profile rats at BL, day 3, day 10 , and day $17[(0.580 \pm 0.109,0.619 \pm 0.064, p>0.05)$, $(0.981 \pm 0.507,1.054 \pm 0.317, \mathrm{p}>0.05),(0.767 \pm 0.369$, $1.074 \pm 0.352, \mathrm{p}>0.05),(0.767 \pm 0.369,0.825 \pm 0.249, \mathrm{p}>0.05)]$ (Graph 3).

Contralateral side: No significance was found between low and high EIH rats at BL, day 3, day 10, and day 17 postsurgery $(0.580 \pm 0.109,0.669 \pm 0.099, \mathrm{p}>0.05),(0.381 \pm 0.191$,

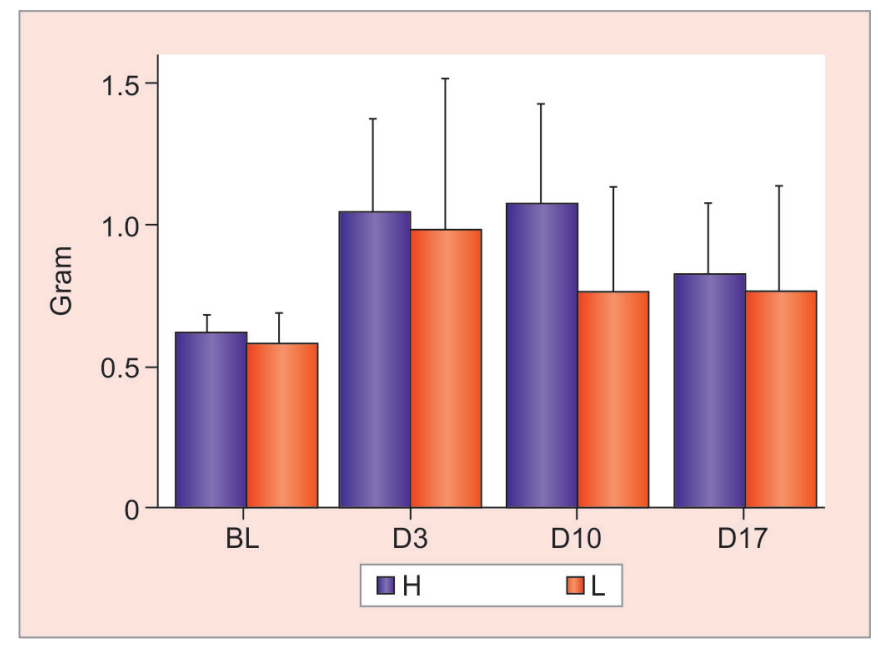

Graph 3: Mechanical allodynia response in grams: Ipsilateral side, $\mathrm{H}=$ high $\mathrm{ElH}$ group, $\mathrm{L}=$ low $\mathrm{EIH}$ group, $\mathrm{BL}=$ baseline, $\mathrm{D} 3=$ day 3 , $\mathrm{D} 10=$ day $10, \mathrm{D} 17=$ day 17

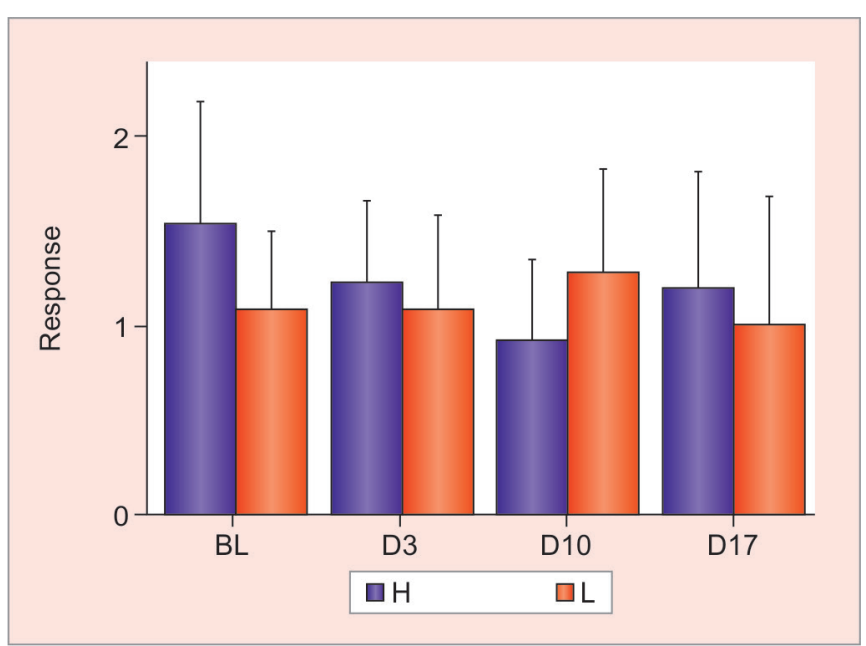

Graph 2: Mechanical hyperalgesia response: Contralateral side, $\mathrm{H}=$ high ElH group, $\mathrm{L}=$ low ElH group, $\mathrm{BL}=$ baseline, $\mathrm{D} 3=$ day 3 , $\mathrm{D} 10=$ day $10, \mathrm{D} 17=$ day 17

$0.581 \pm 0.177, \mathrm{p}>0.05),(0.767 \pm 0.369,0.568 \pm 0.239, \mathrm{p}>0.05)$, $(0.767 \pm 0.369,0.589 \pm 0.154, \mathrm{p}>0.05)($ Graph 4$)$.

\section{DISCUSSION}

The most important finding of the study was that the pinprick behavioral assay was able to predict chronic pain development in the area of the trigeminal nerve distribution using EIH profile measured at the hind paw of the rat. The impact of exercise on pain response is termed as EIH. Exercise can play a role in pain modulation and has been used in the past to determine conditioned pain modulation. ${ }^{22,23}$ Previous studies have shown that pain thresholds increase after physical activities for both mechanical ${ }^{9,18,24}$ and thermal ${ }^{25}$ stimuli, and after isometric muscle contraction, there is increased generalized pressure pain thresholds. ${ }^{12,24}$

The exact mechanism of EIH is still unknown; however, there is different suggested hypothesis believed

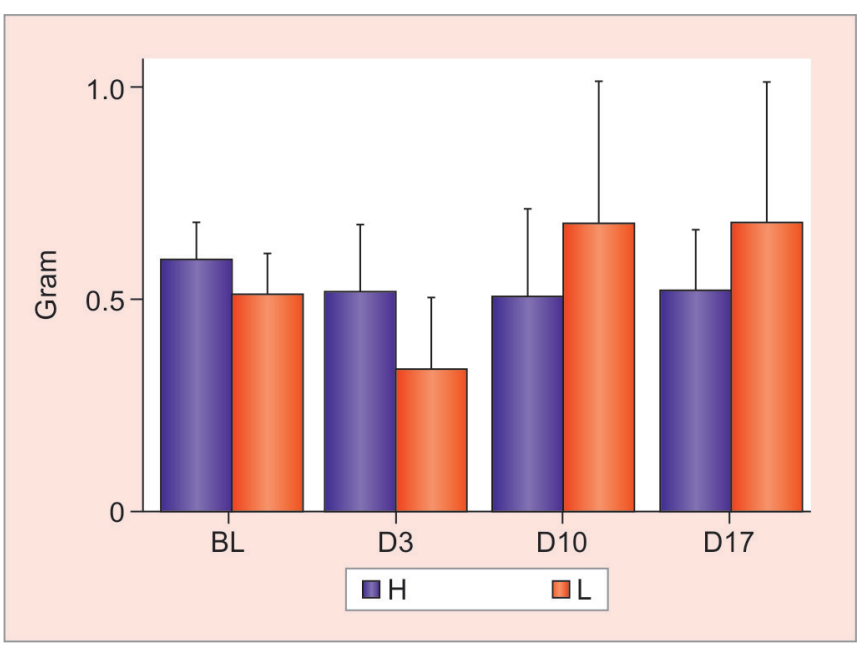

Graph 4: Mechanical allodynia response in grams: Contralateral side, $\mathrm{H}=$ high ElH group, $\mathrm{L}=$ low EIH group, $\mathrm{BL}=$ baseline, $\mathrm{D} 3=$ day $3, \mathrm{D} 10=$ day $10, \mathrm{D} 17=$ day 17 
to activate the inhibitory modulating system through different systems and mechanisms. One among them is the endogeneous opioid system and release of peripheral and central neurotransmitters including beta-endorphins that play an important role. ${ }^{26,27}$ Other mechanisms that may activate inhibitory modulating system are enhancement of inhibitory neurotransmitters, such as serotonin and norepinephrine ${ }^{11}$ interactions with the cardiovascular system, ${ }^{28}$ and the adenosinergic system involvement. ${ }^{16}$

Pain threshold has been monitored to be increased consistently after exercise when the painful stimulus is electrical or chemical but less for thermal stimuli. Other causes that may have an effect on EIH are the type of exercise, duration, intensity, and emotional status and stress. ${ }^{29}$ Nowadays, exercise is considered as an important factor in the management of painful conditions, such as neck pain, fibromyalgia, arthritis, and low back pain. ${ }^{30-33}$

According to a previous study, EIH profile in rats can predict the intensity of tactile allodynia, thermal sensitivity, and mechanical hyperalgesia that developed after nerve injury in sciatic nerve territory. Exercise-induced hypoalgesia profile has also been related to the thermal and mechanical sensitivity in naive rats. In the same study, low EIH rats developed pain in the contralateral side of the surgery, whereas high EIH profile rats did not. This finding suggests that EIH profile may have a role in the development of contralateral (and possible generalized) pain manifestations including trigeminal nervous system. ${ }^{19}$

Using swimming as an exercise for animals has been basically used in previous studies. ${ }^{29}$ It has a lot of limitations, such as stress and temperature variation, which may lead to alteration in the pain response. ${ }^{34,35}$ Using wheel running protocol has shown more accurate results in studies related with pain assessment. ${ }^{19,36}$ However, other models are also available to measure the effect of exercise, such as using low-frequency muscle activation of different muscle groups. ${ }^{37}$

This study is the first using EIH profile in the prediction of developing chronic pain following nerve injury in the trigeminal nerve distribution. The significant finding of this study is the association between the pain intensity following nerve injury and the EIH profile using pinprick needle (mechanical hyperalgesia). Rats with less efficient (low) EIH were more sensitive to and developed significantly greater mechanical hyperalgesia following nerve injury in the affected side, whereas tactile allodynia in the ipsilateral and contralateral side and mechanical hyperalgesia in the contralateral side of surgery were not significant. In a previous study, it was shown that the rats categorized as low EIH have increased significant response to thermal and mechanical stimulants as compared with the high EIH rats, before the nerve injury and after surgery in sciatic nerve, which make the EIH profile predictable for developing chronic pain at the spinal level. ${ }^{19}$

The reason for the differences between high and low EIH rats at BL and following the surgery is still not clear. It may be caused by factors related with reduction after exercise. Inhibiting neurotransmitters in brain and their different levels, such as norepinephrine and serotonin $^{11}$ and activation of the adenosinergic ${ }^{16}$ or the opioid systems in the rat ${ }^{18,26}$ may control the rats' EIH and has the role in pain development after the surgery. Previous studies on athletes have shown that their pain perception differs in comparison with the control group. Athletes had reduced sensitivity to cold and mechanical stimuli, ${ }^{38,39}$ but increased sensitivity to vibration. ${ }^{38}$ The increased response to vibration mediated by A-beta fibers (mechanical allodynia) is an interesting finding, as the vibration detection threshold was the only test that tends to gain more sensation (more sensitive perception). Vibration results in muscle length variations, and then activating low-threshold muscle spindle proprioceptors. ${ }^{40,41}$ That may indicate why there is no significant difference in response with von Frey filaments between high and low EIH profile rats in this study and may suggest pinprick test as a more reliable test than von Frey filaments in the determination of pain development. Other reasons behind the insignificant results with tactile allodynia and mechanical hyperalgesia in contralateral side may be due to the fact that the EIH profile was done in the area of spinal cord system (sciatic nerve) and the nerve injury was established in the face, which is mediated by trigeminal nerve system.

Although the trigeminal system and spinal system have a similarity in some features immediately after nerve injury, later on the two systems go through different pathways. Several evidences and studies showed difference in some neurotransmitter expression following the injuries between the two systems ${ }^{42,43}$ and different changes between spinal nerve injuries and trigeminal nerve injuries in voltage-gated sodium channels. ${ }^{44,45}$

These changes are as follows: (1) The injured spinal nerves develop more spontaneous activity when compared with injured trigeminal nerves, ${ }^{46,47}$ (2) longer period of spontaneous activity in the injured spinal nerve than in the injured trigeminal nerves, ${ }^{46}$ (3) sprouting of the sympathetic nerves develop around the injured spinal nerves, ${ }^{48}$ but not around the injured trigeminal nerves. ${ }^{49,50}$ Therefore, the discovery of EIH profiling models in the trigeminal area may help in the prediction of pain development after nerve injury. Some limitations may include a conservative sample size, so we suggest to increase the sample size in future studies to see if the same trends of results are observed. Second, performing pain behavioral 
assays in the trigeminal area can be challenging and subjective as the results are vastly dependent upon the investigators' experience. More sensitive trigeminal behavioral assays should be used for future studies.

\section{CONCLUSION}

We concluded that the pinprick behavioral assay (mechanical hyperalgesia) was able to predict chronic pain development in the area of the trigeminal nerve distribution using EIH profile measured at the hind paw of the rat. The discovery of EIH profiling models in the trigeminal area may help in the prediction of the pain development after nerve injury more accurately using multiple pain assays.

\section{REFERENCES}

1. Fields HL, Levine JD. Pain - mechanics and management. West J Med 1984 Sep;141(3):347-357.

2. Cole BE. Pain management: classifying, understanding, and treating pain. Hospital Physician 2002 Jun:23-30.

3. Haanpaa M, Attal N, Backonja M, Baron R, Bennett M, Bouhassira D, Cruccu G, Hansson P, Haythornthwaite JA, Iannetti GD, et al. NeuPSIG guidelines on neuropathic pain assessment. Pain 2011 Jan;152(1):14-27.

4. de Miguel M, Kraychete DC, Meyer Nascimento RJ. Chronic pain: cytokines, lymphocytes and chemokines. Inflamm Allergy Drug Targets 2014;13(5):339-349.

5. Hansson PT, Fields HL, Hill RG, Marchettini P, editors. Neuropathic pain: pathophysiology and treatment, progress in pain research and management. Vol. 21. Seattle (WA): IASP Press; 2001. p. 85-106.

6. Assis MR, Silva LE, Alves AM, Pessanha AP, Valim V, Feldman D, Neto TL, Natour J. A randomized controlled trial of deep water running: clinical effectiveness of aquatic exercise to treat fibromyalgia. Arthritis Rheum 2006 Feb 15;55(1):57-65.

7. Wilson WM, Marsden CA. In vivo measurement of extracellular serotonin in the ventral hippocampus during treadmill running. Behav Pharmacol 1996 Jan;7(1):101-104.

8. Young SN. How to increase serotonin in the human brain without drugs. J Psychiatry Neurosci 2007 Nov;32(6):394-399.

9. Whiteside A, Hansen S, Chaudhuri A. Exercise lowers pain threshold in chronic fatigue syndrome. Pain 2004 Jun;109(3):497-499.

10. Hoeger Bement MK, Weyer A, Hartley S, Yoon T, Hunter SK. Fatiguing exercise attenuates pain-induced corticomotor excitability. Neurosci Lett 2009 Mar 13;452(2):209-213.

11. Dietrich A, McDaniel WF. Endocannabinoids and exercise. Br J Sports Med 2004 Oct;38(5):536-541.

12. Kosek E, Lundberg L. Segmental and plurisegmental modulation of pressure pain thresholds during static muscle contractions in healthy individuals. Eur J Pain 2003;7(3):251-258.

13. Kuphal KE, Fibuch EE, Taylor BK. Extended swimming exercise reduces inflammatory and peripheral neuropathic pain in rodents. J Pain 2007 Dec;8(12):989-997.

14. Chen YW, Li YT, Chen YC, Li ZY, Hung CH. Exercise training attenuates neuropathic pain and cytokine expression after chronic constriction injury of rat sciatic nerve. Anesth Analg 2012 Jun;114(6):1330-1337.
15. Shen J, Fox LE, Cheng J. Swim therapy reduces mechanical allodynia and thermal hyperalgesia induced by chronic constriction nerve injury in rats. Pain Med 2013 Apr;14(4):516-525.

16. Martins DF, Mazzardo-Martins L, Soldi F, Stramosk J, Piovezan AP, Santos AR. High-intensity swimming exercise reduces neuropathic pain in an animal model of complex regional pain syndrome type I: evidence for a role of the adenosinergic system. Neuroscience 2013 Mar 27;234:69-76.

17. Sluka KA, O’Donnell JM, Danielson J, Rasmussen LA. Regular physical activity prevents development of chronic pain and activation of central neurons. J Appl Physiol (1985) 2013 Mar 15;114(6):725-733.

18. Hoffman MD, Shepanski MA, Mackenzie SP, Clifford PS. Experimentally induced pain perception is acutely reduced by aerobic exercise in people with chronic low back pain. J Rehabil Res Dev 2005 Mar-Apr;42(2):183-190.

19. Khan J, Benavent V, Korczeniewska OA, Benoliel R, Eliav E. Exercise-induced hypoalgesia profile in rats predicts neuropathic pain intensity induced by sciatic nerve constriction injury. J Pain 2014 Nov;15(11):1179-1189.

20. Imamura $Y$, Kawamoto $H$, Nakanishi O. Characterization of heat-hyperalgesia in an experimental trigeminal neuropathy in rats. Exp Brain Res 1997 Aug;116(1):97-103.

21. Kernisant M, Gear RW, Jasmin L, Vit JP, Ohara PT. Chronic constriction injury of the infraorbital nerve in the rat using modified syringe needle. J Neurosci Methods 2008 Jul 15;172(1):43-47.

22. Lannersten L, Kosek E. Dysfunction of endogenous pain inhibition during exercise with painful muscles in patients with shoulder myalgia and fibromyalgia. Pain 2010 Oct;151(1):77-86.

23. Black J, Chesher GB, Starmer GA, Egger G. The painlessness of the long distance runner. Med J Aust 1979 Jun 2;1(11): 522-523.

24. Hoeger Bement MK, Dicapo J, Rasiarmos R, Hunter SK. Dose response of isometric contractions on pain perception in healthy adults. Med Sci Sports Exerc 2008 Nov;40(11): 1880-1889.

25. Staud R, Robinson ME, Vierck CJ Jr, Price DD. Diffuse noxious inhibitory controls (DNIC) attenuate temporal summation of second pain in normal males but not in normal females or fibromyalgia patients. Pain 2003;101(1-2):167-174.

26. Bement MK, Sluka KA. Low-intensity exercise reverses chronic muscle pain in the rat in a naloxone-dependent manner. Arch Phys Med Rehabil 2005 Sep;86(9):1736-1740.

27. Stagg NJ, Mata HP, Ibrahim MM, Henriksen EJ, Porreca F, Vanderah TW, Philip Malan T Jr. Regular exercise reverses sensory hypersensitivity in a rat neuropathic pain model: role of endogenous opioids. Anesthesiology 2011 Apr;114(4): 940-948.

28. Lovick TA. Integrated activity of cardiovascular and pain regulatory systems: role in adaptive behavioural responses. Prog Neurobiol 1993 May;40(5):631-644.

29. Koltyn KF. Analgesia following exercise: a review. Sports Med 2000 Feb;29(2):85-98.

30. Brosseau L, Wells GA, Tugwell P, Egan M, Wilson KG, Dubouloz CJ, Casimiro L, Robinson VA, McGowan J, Busch A, et al. Ottawa Panel evidence-based clinical practice guidelines for strengthening exercises in the management of fibromyalgia: part 2. Phys Ther 2008 Jul;88(7):873-886.

31. Jansen MJ, Viechtbauer W, Lenssen AF, Hendriks EJ, de Bie RA. Strength training alone, exercise therapy alone, and exercise therapy with passive manual mobilisation each reduce pain 
and disability in people with knee osteoarthritis: a systematic review. J Physiother 2011;57(1):11-20.

32. Stewart MJ, Maher CG, Refshauge KM, Herbert RD, Bogduk N, Nicholas M. Randomized controlled trial of exercise for chronic whiplash-associated disorders. Pain 2007 Mar;128(1-2):59-68.

33. van Middelkoop M, Rubinstein SM, Verhagen AP, Ostelo RW, Koes BW, van Tulder MW. Exercise therapy for chronic nonspecific low-back pain. Best Pract Res Clin Rheumatol 2010 Apr;24(2):193-204.

34. Willow M, Carmody J, Carroll P. The effects of swimming in mice on pain perception and sleeping time in response to hypnotic drugs. Life Sci 1980 Jan 21;26(3):219-224.

35. Christie MJ, Chesher GB, Bird KD. The correlation between swim-stress induced antinociception and $[3 \mathrm{H}]$ leu-enkephalin binding to brain homogenates in mice. Pharmacol Biochem Behav 1981 Dec;15(6):853-857.

36. Kandasamy R, Calsbeek JJ, Morgan MM. Home cage wheel running is an objective and clinically relevant method to assess inflammatory pain in male and female rats. J Neurosci Methods 2016 Apr 1;263:115-122.

37. Hoffmann P, Skarphedinsson JO, Delle M, Thoren P. Electrical stimulation of the gastrocnemius muscle in the spontaneously hypertensive rat increases the pain threshold: role of different serotonergic receptors. Acta Physiol Scand 1990 Feb;138(2):125-131.

38. Tesarz J, Gerhardt A, Schommer K, Treede RD, Eich W. Alterations in endogenous pain modulation in endurance athletes: an experimental study using quantitative sensory testing and the cold-pressor task. Pain 2013 Jul;154(7):1022-1029.

39. Janal MN, Glusman M, Kuhl JP, Clark WC. Are runners stoical? An examination of pain sensitivity in habitual runners and normally active controls. Pain 1994 Jul;58(1): 109-116.

40. Fallon JB, Macefield VG. Vibration sensitivity of human muscle spindles and Golgi tendon organs. Muscle Nerve 2007 Jul;36(1):21-29.
41. Verschueren SM, Cordo PJ, Swinnen SP. Representation of wrist joint kinematics by the ensemble of muscle spindles from synergistic muscles. J Neurophysiol 1998 May;79(5):2265-2276.

42. Kordeli E, Lambert S, Bennett V. Ankyrin G. A new ankyrin gene with neural-specific isoforms localized at the axonal initial segment and node of Ranvier. J Biol Chem 1995 Feb 3;270(5):2352-2359.

43. Rasband MN, Peles E, Trimmer JS, Levinson SR, Lux SE, Shrager P. Dependence of nodal sodium channel clustering on paranodal axoglial contact in the developing CNS. J Neurosci 1999 Sep 1;19(17):7516-7528.

44. Bongenhielm U, Nosrat CA, Nosrat I, Eriksson J, Fjell J, Fried K. Expression of sodium channel SNS/PN3 and ankyrin $(\mathrm{G})$ mRNAs in the trigeminal ganglion after inferior alveolar nerve injury in the rat. Exp Neurol 2000 Aug;164(2):384-395.

45. Coward K, Plumpton C, Facer P, Birch R, Carlstedt T, Tate S, Bountra C, Anand P. Immunolocalization of SNS/PN3 and NaN/SNS2 sodium channels in human pain states. Pain. 2000 Mar;85(1-2):41-50.

46. Tal M, Devor M. Ectopic discharge in injured nerves: comparison of trigeminal and somatic afferents. Brain Res 1992 May 1;579(1):148-151.

47. Cherkas PS, Huang TY, Pannicke T, Tal M, Reichenbach A, Hanani M. The effects of axotomy on neurons and satellite glial cells in mouse trigeminal ganglion. Pain 2004 Jul;110 (1-2):290-298

48. McLachlan EM, Janig W, Devor M, Michaelis M. Peripheral nerve injury triggers noradrenergic sprouting within dorsal root ganglia. Nature 1993 Jun 10;363(6429):543-546.

49. Bongenhielm U, Boissonade FM, Westermark A, Robinson PP, Fried K. Sympathetic nerve sprouting fails to occur in the trigeminal ganglion after peripheral nerve injury in the rat. Pain 1999 Sep;82(3):283-288.

50. Benoliel R, Eliav E, Tal M. No sympathetic nerve sprouting in rat trigeminal ganglion following painful and nonpainful infraorbital nerve neuropathy. Neurosci Lett 2001 Jan 19;297(3):151-154. 